\title{
Evaluating Medication Use for Continuous Quality Improvement in Diabetes Care
}

\author{
DAVID P. NAU, PhD
}

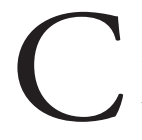
ontinuous quality improvement (CQI) is the structured organizational process for involving personnel in planning and executing a continuous flow of improvements to provide services that meet or exceed expectations. ${ }^{1}$ It is integral to the long-term success of managed care organizations (MCOs). Many purchasers of health care, along with accrediting bodies, have encouraged the adoption of the CQI philosophy and its tools. The hope is that CQI will lead to more efficient care as well as better health for members enrolled in health plans. The value of CQI is generally accepted, and consistent with the philosophy of CQI, the methods for measuring quality are undergoing constant evolution.

The most common method for evaluating the quality of MCOs is the Health Plan Employer Data and Information Set (HEDIS) from the National Committee for Quality Assurance (NCQA). HEDIS includes performance indicators related to health promotion and the effectiveness of care for selected diseases. These performance indicators offer insight to the quality of care provided by MCOs. The number of performance indicators within HEDIS has expanded over the past several years, and the technical specifications for the indicators have been refined.

Reported results and trends in the performance indicators over the past several years suggest that NCQA has been successful in stimulating quality improvement in $\mathrm{MCOs}^{2,3}$ Nonetheless, HEDIS does have limitations. Kerr and colleagues have pointed out several pitfalls in the use of HEDIS for quality improvement. ${ }^{4}$ These pitfalls include the lack of a strong link between many process-oriented measures and outcomes, intermediate outcomes-oriented measures that are not linked to actionable processes, measures that do not target those at highest-risk, the lack of severity adjustment for outcomes measures, and the inability of the measurement system to allow for patient exceptions. These pitfalls in the measurement system limit the utility of HEDIS for quality improvement efforts. Ideally, the current indicators in HEDIS would be supplemented by additional measures of quality that help to pinpoint specific health care processes that need improvement.

Author

DAVID P. NAU, PhD, is an Assistant Professor, Department of Social and Administrative Sciences, Center for Medication Use, Policy and Economics, University of Michigan College of Pharmacy, Ann Arbor, Michigan.

AUTHOR CORRESPONDENCE: David P. Nau,PhD: E-mail:dnau@umich.edu

Copyright $\odot$ 2002, Academy of Managed Care Pharmacy. All rights reserved.
Medications are the mainstay of therapy for many chronic diseases. Thus, the medication-use process should be a focal point for CQI initiatives in populations that have diseases such as asthma, diabetes, chronic heart failure, dyslipidemia, or hypertension. While the current HEDIS indicator set does contain a few medication-specific indicators (e.g., beta-blocker treatment following a myocardial infarction and appropriate medications for the treatment of persistent asthma and depression), there are still relatively few indicators targeted specifically on the medicationuse system. One disease state that lacks well-established medication-specific indicators is diabetes mellitus.

\section{Quality Measurement for Diabetes}

For MCOs, the standard for quality measurement in diabetes has been the HEDIS diabetes indicator set. Since 1999, the HEDIS Comprehensive Diabetes Care indicator set has estimated the percentage of health plan members with type 1 and type 2 diabetes who were 18-75 years old and during the measurement year had (1) a glycosylated hemoglobin (HbAlc) test; (2) poorly controlled HbAlc (HbAlc>9.5\%); (3) a cholesterol (LDL-c) test; (4) an LDL-c level less than $130 \mathrm{mg} / \mathrm{dl}$; (5) a microalbumin test; and (6) a dilated eye exam. ${ }^{3}$ In 2001, NCQA partnered with the American Medical Association and the Joint Commission on Accreditation of Healthcare Organizations to develop a consensus statement on "Coordinated Performance Measurement for the Management of Adult Diabetes." This consensus statement proposes quality measures that go beyond the HEDIS reporting set for diabetes. Specifically, it adds indicators related to blood pressure measurement and control, foot exams, office visits, and influenza immunizations.

The Veterans Affairs Quality Enhancement Research Initiative is currently testing several indicators for diabetes mellitus, including 2 measures that target the appropriate use of medications for treatment of dyslipidemia and hypertension in persons with diabetes. ${ }^{4,6}$ The indicators capture whether appropriate initiation or adjustments of drug therapy occurred for persons with diabetes who had elevated LDL and/or elevated blood pressure. These new measures should enhance the quality improvement efforts of the Department of Veterans Affairs by examining specific provider behaviors that are tightly linked with patient outcomes.

Although advancements have been made in quality measurement for diabetes mellitus, there are still relatively few indicators that focus on the use of medications in this population. Although this is a shortcoming of our quality measurement efforts, there may be several reasons for the failure to target medication use in diabetes. For example, initial indicator devel- 
Evaluating Medication Use for Continuous Quality Improvement in Diabetes Care

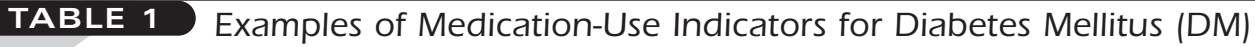

Indicator

Initiate/Adjust Therapy

Definition

Population

Caveats

- Diabetes Proportion of patients who had an HbAlc reading above $9.5 \%$ in the reporting year and who had initiation or intensification of drug therapy within 3 months of the elevated HbAlc.

- Hypertension* Proportion of patients with DM who had 2 blood pressure readings > 140/90 $\mathrm{mmHg}$ in the reporting year and who had an initiation or intensification of drug therapy within 3 months of the elevated readings.

-Dyslipidemia*

Proportion of patients with DM who had an LDL level $>130 \mathrm{mg} / \mathrm{dl}$ in the reporting year and who had an initiation or intensification of drug therapy within 3 months of the elevated reading.
Patients with an HbAlc reading above $9.5 \%$ during the reporting year.

Patients with DM who had 2 blood pressure readings > $140 / 90 \mathrm{mmHg}$ in the reporting year.

Patients with DM who had an LDL level $>130 \mathrm{mg} / \mathrm{dl}$ in the reporting year

\begin{abstract}
These indicators do not take into account the other modalities used to treat DM, hypertension or dyslipidemia (e.g., diet, exercise).

These indicators would not include patients with DM who have not had a measurement of HbAlc, blood pressure, or lipids.
\end{abstract}

\footnotetext{
Adherence to Therapy Using administrative claims: The proportion of patients who obtained a supply of medication that would cover at least $90 \%$ of days in the assessment period.

Using self-reported data: The proportion of patients who reported taking at least $90 \%$ of oral antidiabetic drug doses as directed.

Monitoring for ADR $\dagger$

- $\mathrm{TZD}$ ₹

- Metformin

The proportion of patients dispensed a TZD who had a baseline and follow-up test of serum ALT.

The proportion of patients dispensed metformin who had a baseline and follow-up test of renal function (serum creatinine)

* Adapted from Kerr EA, Krein SL, Vijan S, et al. Am J Managed Care. 2001;7:1033-43.

$\dagger A D R=$ Adverse drug reaction.

\#ZD = thiazolidinediones (e.g., rosiglitazone, pioglitazone).
}

Patients with an active prescription for oral diabetes medication throughout the reporting year.

\author{
For patients on multi-drug \\ therapy for diabetes, the first \\ oral antidiabetic agent listed in \\ the drug regimen could be \\ used for analyses in lieu of \\ more complex analyses involv- \\ ing all antidiabetic agents.
}

Patients prescribed a TZD for at least 2 months

Patients prescribed metformin for at least 2 months. opment efforts were often focused on whether appropriate screening and monitoring for conditions occurred (e.g., was the cholesterol and HbAlc checked?). This may have been an appropriate place to start our indicator development efforts since one can't effectively manage a clinical problem if one doesn't know whether the problem exists. However, measuring $\mathrm{HbAlc}$ is not enough. We must be making appropriate modifications of therapy to ensure that the HbAlc measurement leads to positive outcomes.

There are also challenges to be overcome in data collection when focusing on medication management. Not all managed care patients have a prescription benefit. This limits the feasibility of tracking prescription claims for CQI. Even when prescription claims data are available, the data are often separate from medical claims and need to be merged with other data to provide useful information. Finally, the drug therapy of patients with diabetes is complex, and thus, no simple standard can be applied for which drug is best for each patient.

Despite the difficulties in creating valid and reliable indicators for diabetes, it may still be useful for a managed care organization to evaluate the quality of medication management for their members with diabetes. Patients with diabetes often have 
comorbid conditions such as dyslipidemia, hypertension, and depression. All of these conditions are managed, in part, through the use of drug therapy. Thus, the appropriate management of medication is highly important for achieving optimal outcomes in this population. ${ }^{7,8}$ The development of medication-use indicators could facilitate CQI efforts for diabetes by identifying specific prescribing, monitoring, or self-management behaviors that are in need of improvement.

\section{Medication-use Indicators for Diabetes}

There are numerous medication-use indicators that could be constructed for persons with diabetes mellitus. Ideally, the indicators would address various aspects of the medication-use system such as initiation of therapy, selection of drug products, monitoring of therapy, adherence to therapy, and adjustments of therapy. ${ }^{9}$ Several examples of indicators are shown in Table 1.

\section{Initiation/Adjustment of Therapy}

The first steps for practitioners in the medication-use system are to diagnose the medical problems exhibited by their patients and to prescribe drugs based upon the diagnoses. Although current HEDIS indicators for diabetes are useful for determining whether practitioners are screening patients for specified problems (e.g., was the HbAlc checked?), they do not provide insight on whether appropriate drug therapy was prescribed in response to the identified problems or adjusted when necessary to achieve the therapeutic goals.

Kerr and colleagues have proposed measures that would help to evaluate whether drug therapy was initiated or adjusted in response to elevated LDL or blood pressure in persons with diabetes mellitus. ${ }^{4}$ These measures are currently being tested in the Veterans Affairs health system. Adaptations of these measures are represented in Table 1. Selecting the cut-point for each of these indicators is a crucial issue. In one indicator proposed by Kerr and colleagues, an LDL of $130 \mathrm{mg} / \mathrm{dl}$ is used as the cutpoint. Patients above this point are identified as being at high risk for cardiovascular events and are thus included in the indicator. This cut-point is consistent with the parameters established in other HEDIS indicators using LDL. ${ }^{3}$

Additionally, the American Diabetes Association recommends that drug therapy be initiated in diabetes patients (who do not have preexisting coronary heart disease) when the LDL has exceeded $130 \mathrm{mg} / \mathrm{dl} . .^{10}$ For diabetes patients with preexisting coronary heart disease, the ADA recommends that drug therapy be initiated along with medical nutrition therapy when the LDL exceeds $100 \mathrm{mg} / \mathrm{dl}$. The therapeutic goal for all diabetes patients being treated for dyslipidemia is an $\mathrm{LDL}<100 \mathrm{mg} / \mathrm{dl}$. Although it may be tempting to construct an indicator that identifies the proportion of patients who have not reached the therapeutic goal of $\mathrm{LDL}<100 \mathrm{mg} / \mathrm{dl}$, this more stringent cut-point may lead to the inappropriate conclusions about the quality of medication use. For example, it may be appropri- ate for a clinician to choose not to increase the intensity of pharmacological therapy for a diabetes patient with $\mathrm{LDL}=102 \mathrm{mg} / \mathrm{dl}$. However, it would be more difficult to justify the failure to modify therapy in a diabetes patient with $\mathrm{LDL}=132 \mathrm{mg} / \mathrm{dl}$. Thus, the cut-point of $130 \mathrm{mg} / \mathrm{dl}$ is perhaps less likely to lead to inappropriate categorizations of the quality of therapy.

The logic found in Kerr et al.'s indicators could be applied to the evaluation of oral antidiabetic drug therapy. If the HbAlc is significantly elevated $(>9.5 \%)$, then an initiation or adjustment of drug therapy will likely be required to help the patient achieve the therapeutic goal for HbAlc of $6.5 \% .{ }^{11}$ The cut-point of $\mathrm{HbAlc}=9.5 \%$ is consistent with existing HEDIS indicators and other quality measurement systems., ${ }^{3,5}$ Although diet and exercise can also be used to lower HbAlc, most patients with an HbAlc above $9.5 \%$ will require drug therapy. For patients who are already taking antidiabetic medications, an $\mathrm{HbAlc}$ above 9.5\% should signal the need for an adjustment in therapy.

Data for constructing the indicators could be obtained through a combination of administrative claims and chart review as specified for the existing HEDIS diabetes indicators. Since chart reviewers are already extracting data on HbAlc, LDL, and blood pressure, they could also determine whether the drug therapy was adjusted within 3 months of the tests. While this would lengthen the time required to complete the chart review, the value of the data may exceed the additional cost for chart review. In a health care system with electronic laboratory data or a system-wide electronic medical record, the electronic medical/laboratory data could be compared to the prescription claims data to determine if a change in medication occurred subsequent to the laboratory test or blood pressure assessment.

\section{Adherence to Therapy}

Oral medications are one of the key factors to glycemic control for many persons with type 2 diabetes. However, many persons find it difficult to adhere to their medication regimen for long periods of time. A recent study found that fewer than $15 \%$ of diabetes patients on single-drug therapy maintained ideal adherence throughout a one-year period, while patients on multi-drug therapy for diabetes experienced even lower levels of persistence and adherence. ${ }^{12}$ Considering that many patients with type 2 diabetes have other comorbidities (e.g., dyslipidemia, hypertension), the drug regimen for these patients is often very complex and adherence is even more challenging. Many MCOs have implemented disease management strategies that include efforts to enhance patients' medication regimen adherence. Because of the high costs of disease management programs and the potentially higher costs of nonadherence, it is important to evaluate whether these programs have led to improvements in patients' persistence and adherence.

There are many ways in which medication regimen adherence has been measured. ${ }^{13}$ The methods that would be most fea- 
sible for a managed care organization entail an analysis of prescription claims or a survey of patients. Steiner and Prochazka described several ways in which adherence rates can be calculated from prescription claims data. ${ }^{14}$ However, all of these methods rely on having complete and accurate claims data for prescription drugs. While most prescription drug claims are accurate, the quality of the data may not be sufficient to allow valid comparisons between health plans. ${ }^{15}$ Additionally, some managed care enrollees may not have complete prescription drug benefits or may be obtaining some medications outside of the prescription benefit. For patients with diabetes, it would also be difficult to estimate an adherence rate for persons using insulin since many patients may adjust their dose based upon their changing need for insulin. Nonetheless, it may be useful to determine whether patients have been obtaining refills of oral medications or blood glucose test strips. The trend in the refill rate for the diabetes population could be tracked over time to determine whether the implementation of new services leads to improvements in medication/strip refill adherence.

Surveys of patients can also be used to measure medication regimen adherence. A number of different self-report measures can be used. ${ }^{13}$ Some of these measures ask patients to report the number of missed doses over a specified time frame, while others may use visual analog scales or multidimensional scales. ${ }^{13,16}$ Some measures are scored as continuous variables (i.e., percent of doses taken) while others convert the data to a dichotomous scale (adherent versus nonadherent). It is also important to recognize that different self-report measures may provide different results when applied to the same patients. ${ }^{16}$ Thus, comparisons of adherence rates are only appropriate when using identical adherence measures.

An advantage of surveys is that one can also ask patients about the barriers that they encounter in using their medications. This may help to pinpoint common problems encountered by your members with diabetes. Services could then be developed or modified to meet the needs of this population. Repeating the survey on a regular basis could then inform you about whether service modifications actually diminished the barriers to adherence and improved medication use. Although many practitioners may suspect that nonadherent patients would not be truthful on a survey, the existing literature shows that confidential surveys may be reasonably accurate when estimating the adherence rate within a population. ${ }^{13}$ Thus, the advantages of patient surveys may outweigh their shortcomings.

\section{Monitoring of Drug Therapy}

One of the most important elements of the medication-use system is the monitoring of drug therapy. Monitoring allows us to determine if the patient is achieving the therapeutic goal and to identify adverse drug reactions. The monitoring of patients' progress toward therapeutic goals is already captured by many of the HEDIS diabetes indicators. However, there are no indica- tors related to monitoring for adverse reactions. Because of the possibility of significant, albeit rare, adverse drug reactions, the manufacturers of metformin have recommended the recurrent monitoring of renal function and the manufacturers of the thiazolidinedione (TZD) class of drugs have developed guidelines for the monitoring of hepatic function. ${ }^{17-19}$ Because the occurrence of these problems is rare, it is appropriate to weigh the cost versus benefit of requiring all MCOs to construct indicators regarding the incidence of the adverse events. It could be argued that practitioners should be following the recommended monitoring guidelines for these drugs and that their adherence to the monitoring guidelines is worthy of attention. In health care systems with electronic medical records, it may be cost-effective to measure practitioner adherence to the drug monitoring guidelines for metformin and TZDs.

\section{Other Potential Indicators}

Many other indicators could be selected for the evaluation of the medication use system for diabetes depending on the particular concerns of the MCO. For example, one could measure the proportion of diabetes patients with hypertension who receive an angiotensin-converting enzyme inhibitor (ACEI) or angiotensin receptor blocker (ARB). The ACEI/ARB classes of drugs have been recommended by the American Diabetes Association as first-line therapy for the treatment of hypertension in persons with diabetes due to the ability of these drugs to slow the development or progression of diabetic nephropathy. ${ }^{20}$ Although an ACEI or ARB should be considered for every person with diabetes and hypertension, these drugs may not be appropriate for some patients. In order to determine whether care for an individual patient was appropriate, information from the medical record would be required. Consequently, examining only the trend in claims for antihypertensive medications has its limitations. A trend toward increased use of ACEI/ARBs in patients with diabetes and hypertension may indicate improved quality of medication prescribing; however, it is difficult to know the "ideal" rate of ACEI/ARB utilization without detailed, patient-specific, medical information.

Even more indicators could be adapted from the Academy of Managed Care Pharmacy Catalog of Pharmacy Quality Indicators. ${ }^{21}$ While the indicators in the catalog are not disease-specific, many of them could be adapted to medication utilization patterns in diabetes.

\section{Using the Indicators for COI}

Indicators should be a part of a quality improvement program wherein they help to identify areas in need of improvement. They should be selected based upon both the value of the information provided and the feasibility of data collection. ${ }^{1,22}$ It may be wise to gradually test and adopt new indicators rather than overwhelm an MCO's staff with a slew of new indicators. Consequently, each organization should prioritize its potential 
indicators and select a small number of indicators that will be most useful, while not significantly adding to the burden of data collection. Of the sample indicators listed in Table 1, the most useful indicators may be those related to the initiation/adjustment of therapy. These indicators reflect a highly important element of care and provide "actionable" information in that they identify a specific provider behavior that can be modified. Additionally, collection of data for these indicators could be integrated with the existing HEDIS data collection.

A diabetes quality improvement team within each $\mathrm{MCO}$ could respond to the indicator data by identifying the root causes for suboptimal performance and formulating plans for improvement. Potential strategies for improving performance might include (1) the creation or modification of guidelines for the use of diabetes medications, (2) educating providers and patients on the new guidelines, (3) providing performance feedback to individual practitioners, or (4) sending electronic prompts to physicians regarding the potential need for therapy adjustments when lab values exceed predetermined thresholds. By having repeated measurements of indicator data, you can determine whether CQI efforts have led to actual improvements.

CQI has been successfully applied in MCOs. ${ }^{23,24}$ A collaborative effort of physicians, nurses, pharmacists, patients, data analysts, and other MCO staff can identify opportunities for improvement, formulate and implement plans for improvement, and evaluate the impact of those plans. However, failure to assess key elements of the plan (e.g., medication use) may miss potential explanations for the success or failure of the QI plan. Without valid and actionable data, CQI efforts are hampered.

The introduction of HEDIS has led to advances in improvements in the quality of care for persons with diabetes., ${ }^{2,3}$ These improvements have mainly pertained to the increased screening and monitoring patients of patients with diabetes. Now it is time to move beyond the initial achievements of HEDIS to examine the ways providers and MCOs respond to the monitoring data. Have adjustments been made in therapy for patients for whom $\mathrm{HbAlc}$ is significantly elevated? Has treatment been initiated for patients with elevated blood pressure? Asking questions like these should help to "close the loop" in the medication use system as more attention is focused on the need to continually manage drug therapy to help patients achieve their therapeutic goals.

\section{DISCLOSURES}

No outside funding supported this study. The author does not have financial disclosures or a conflict of interest.

\section{REFERENCES}

1. McLaughlin CP, Kaluzny AD. Continuous Quality Improvement in Health Care: Theory, Implementation and Applications. 2nd ed. Gaithersburg, MD: Aspen Publishers; 1999

2. National Committee for Quality Assurance. Quality dividends, 2001.
Available at: www.ncqa.org.

3. National Committee for Quality Assurance. The state of managed care quality, 2001. Available at: www.ncqa.org.

4. Kerr EA, Krein SL, Vijan S, et al. Avoiding pitfalls in chronic disease quality measurement: a case for the next generation of technical quality measures. Am J Managed Care. 2001;7:1033-43.

5. American Medical Association, Joint Commission on Accreditation of Healthcare Organizations, National Committee for Quality Assurance.

Coordinated performance measurement for the management of adult diabetes: April 2001. Available at: www.ama-assn.org/ama/pub/category/3798.html.

6. Veterans Administration. Quality enhancement research initiatitive. Available at: www.va.gov/annarbor-hsrd/QUERI-DMhome.html.

7. The Diabetes Control and Complications Trial Research Group. The effect of intensive treatment of diabetes on the development and progression of long-term complications in insulin-dependent diabetes mellitus. N Engl J Med. 1993;329:977-86

8. UK Prospective Diabetes Study Group. Intensive blood-glucose control with sulphonylureas or insulin compared with conventional treatment and risk of complications in patients with type 2 diabetes (UKPDS 33). Lancet. $1998 \cdot 352: 837-53$

9. Hepler CD, Grainger-Rousseau TJ. Pharmaceutical care versus traditional drug treatment. Drugs. 1995;49:1-10.

10. American Diabetes Association. Management of dyslipidemia in adults with diabetes. Diabetes Care. 2002;25:S74-S77.

11. American Association of Clinical Endocrinologists. Medical guidelines for the management of diabetes mellitus, 2002. Available at:

www.aace.com/clin/guidelines/diabetes_2002.pdf.

12. Dailey G, Kim MS, Lian JF. Patient compliance and persistence with antihyperglycemic drug regimens: evaluation of a Medicaid patient opulation with type 2 diabetes mellitus. Clin Ther. 2001;23:1311-20.

13. Farmer KC. Methods for measuring and monitoring medication regimen adherence in clinical trials and clinical practice. Clin Ther. 1999;21:1074-90.

14. Steiner JF, Prochazka AV. The assessment of refill compliance using pharmacy records: Methods, validity, and applications. J Clin Epidemiol. 1997;50:105-16.

15. Farris KB, Kaplan B, Kirking DM. Examination of days supply in computerized prescription claims. J Pharmacoepidemiol. 1994;3:63-76.

16. Gao X, Nau DP. Congruence of 3 self-report measures of medication adherence among HIV patients. Ann Pharmacother. 2000;34:1117-22.

17. Bristol-Myers Squibb. Metformin prescribing information. Available at: www.glucophagexr.com

18. Takeda Pharmaceuticals America. Actos prescribing information. Available at: www.actos.com.

19. GlaxoSmithKline. Avandia prescribing information. Available at: www.avandia.com.

20. American Diabetes Association. Treatment of hypertension in adults with diabetes. Diabetes Care. 2002;25:S71-S73.

21. Academy of Managed Care Pharmacy. Catalog of Pharmacy Quality Indicators. Available at: http://www.amcp.org/publications/index_publications.asp. Accessed: August 13, 2002.

22. Angaran DM. Selecting, developing, and evaluating indicators. Am J Hosp Pharm. 1991;48:1931-37.

23. Petitti DB, Dudi J, Contreras R, et al. Evaluation of the effect of performance monitoring and feedback on care process, utilization, and outcome. Diabetes Care. 2000;23:192-96.

24. Solberg LI, Kottke TE, Brekke ML. CQI in Managed Care. In: McLaughlin CP, Kaluzny AD, ed. Continuous Quality Improvement in Health Care: Theory,

Implications and Applications. Gaithersburg, MD: Aspen Publishers; 1999:298-322. 